\title{
Motivação à prática regular de atividade física: um estudo exploratório
}

\author{
Marcos Alencar Abaide Balbinotti \\ Université du Québec à Trois-Rivières \\ Marcus Levi Lopes Barbosa \\ Carlos Adelar Abaide Balbinotti \\ Ricardo Pedrozo Saldanha \\ Universidade Federal do Rio Grande do Sul
}

\begin{abstract}
Resumo
A motivação na perspectiva da Teoria da Autodeterminação (TAD) sugere que uma pessoa pode ser motivada em diferentes níveis. Neste estudo essas diferenças são exploradas através de comparações dos níveis gerais de motivação, observando-se as variáveis "sexo e faixa etária". Uma amostra de 635 praticantes de ambos os sexos e com idades variando de 18 a 55 anos respondeu o "Inventário de Motivação à Prática Regular de Atividade Física e Esportiva” (IMPRAFE-126). Os resultados indicam haver diferença significativa $\left(F_{(2,632)}\right.$ $=7,214 ; p=0,001)$ somente quando controlada a faixa etária. As diferenças foram interpretadas conforme preconiza a TAD e com relação aos estados e tarefas do desenvolvimento.
\end{abstract}

Palavras-chave: motivação; atividade física; estudo exploratório.

\begin{abstract}
Regular practical motivation for physical activity: an exploratory study. Motivation in Self-Determination Theory (SDT) suggests that one may be motivated in different levels. In this study these differences are explored through comparisons of the motivation general levels, taking into account the "gender and age groups" variables. A sample of 635 participants from both gender and ages between 18 and 55 years old answered the Motivation to the Regular Practice of Physical and Sports Activity Inventory (IMPRAFE-126). Results point significant difference $\left(F_{(2,632)}=7,214 ; p=0,001\right)$ only when controlled the age group. Differences were interpreted as recommended by SDT and related to the states and tasks of the development.
\end{abstract}

Keywords: motivation; physical activity; exploratory study.

$\mathrm{E}$ ste estudo de natureza exploratória avaliou os níveis gerais da motivação que levam indivíduos adultos que frequentam academias de ginástica e clubes esportivos à prática regular de atividades físicas e esportivas. Além disso, foi testada a ocorrência de possíveis diferenças significativas nos níveis de motivação. Os dados foram interpretados com base nos pressupostos da Teoria da Autodeterminação (Deci \& Ryan, 1985; Ryan \& Deci, 2000a), considerando as variáveis de controle: sexo e faixa etária. Para tanto, inicialmente, serão apresentados aspectos referentes ao plano teórico relativo à motivação e, em seguida, aqueles referentes ao plano empírico.

\section{A motivação no contexto da teoria da autodetermina- ção de Deci e Ryan}

Elaborada por Deci e Ryan (1985, 2000a), a Teoria da Autodeterminação é amplamente difundida e utilizada em diversas áreas do conhecimento acadêmico (Deci, Ryan, \& Koestner, 1999), dentre os quais o esporte e atividade física
(Barbosa, 2006; Deci \& Olson, 1989; Frederick \& Ryan, 1995). Esta teoria preconiza que um sujeito pode ser motivado em diferentes níveis (intrínseca ou extrinsecamente), ou ainda, ser "amotivado" durante a prática de qualquer atividade.

Quando intrinsecamente motivado, o sujeito ingressa na atividade por sua própria vontade, ou seja, pelo prazer e satisfação do processo de conhecê-la, explorá-la, aprofundála. Atividades intrinsecamente motivadas são comumente associadas ao bem estar psicológico, interesse, alegria e persistência (Ryan \& Deci, 2000b). Autores têm subdividido a motivação intrínseca em 3 tipos: "para saber", "para realizar" e "para experiência". A motivação intrínseca "para saber" ocorre quando se executa uma atividade para satisfazer uma curiosidade, ao mesmo tempo em que se aprende tal atividade; a motivação intrínseca "para realizar" ocorre quando um indivíduo realiza uma atividade pelo prazer de executá-la e a motivação intrínseca "para experiência" ocorre quando um indivíduo realiza uma atividade para experienciar as situações estimulantes inerentes 
à tarefa (Brière, Vallerand, Blais, \& Pelletier, 1995).

A motivação extrínseca, por sua vez, ocorre quando uma atividade é efetuada com outro(s) objetivo(s) que não o(s) inerente(s) à própria atividade (Ryan \& Deci, 2000a). Estes motivos podem variar grandemente em relação ao seu grau de autonomia, criando, basicamente, três categorias desta motivação: 1) a "regulação externa": esta categoria da motivação extrínseca ocorre quando o comportamento é regulado por premiações materiais ou medo de consequências negativas, como críticas sociais (este tipo de motivação pode ser observado no âmbito esportivo quando o treinador impõe punições aos atletas, quando não realizam as tarefas propostas); 2) a "regulação interiorizada": é a categoria que ocorre quando o comportamento é regulado por uma fonte de motivação que, embora inicialmente externa, é internalizada, como comportamentos reforçados por pressões internas como a culpa, ou como a necessidade de ser aceito; c) a 'regulação identificada': é a categoria da motivação extrínseca que ocorre quando um sujeito realiza uma tarefa (ou comportamento), a qual não lhe é permitida a escolha; uma atividade que é considerada como importante de ser realizada, mesmo que não lhe seja interessante.

Há ainda um terceiro tipo de motivação. Trata-se da "amotivação", construção motivacional que pode ser encontrada em indivíduos que ainda não estão adequadamente aptos a identificar um bom motivo para realizar alguma atividade física (Ryan \& Deci, 2000a). No ponto de vista destes indivíduos, a atividade ou não lhes trará nenhum benefício, ou eles não conseguirão realizá-la de modo satisfatório (Brière et al., 1995).

Detalhados os níveis do contínuo motivacional, cabe salientar que a Teoria da Autodeterminação (Ryan \& Deci, 2000a) é uma meta-teoria. Desta maneira, estes níveis de autodeterminação são explicados por cinco subteorias: "Teoria da Avaliação Cognitiva", "Teoria da Integração Orgânica", "Teoria da Orientação Causal”, "Teoria das Necessidades Básicas”, e “Teoria de Orientação de Metas". A Teoria de Orientação de Metas pressupõe que a orientação das metas pessoais em uma atividade explica os níveis de autodeterminação. Este estudo toma como base esta última subteoria. À luz destas diferentes subteorias, destaca-se que há ao menos dois caminhos para medir a motivação: um, é medindo os níveis de autodeterminação, como fez Fernandes e Vasconcelos-Raposo (2005) e outro é medindo os motivos que levam estes sujeitos a esta prática, como fizeram Ryan, Frederick, Lepes, Rubio e Sheldon (1997). Este estudo adota o segundo destes dois caminhos.

\section{A motivação no plano empírico}

Partindo destes pressupostos teóricos, vários pesquisadores, com o intuito de melhor conhecer diversos aspectos sobre os fatores motivacionais, buscaram relacionar a motivação à prática de atividades físicas ao gênero, à idade (Balbinotti \& Capozzoli, 2008; Barbosa, 2006; Castro, 1999; Lores, Murcia, Sanmartín, \& Camacho, 2004), e a outras variáveis (Wang \& Wiese-Bjornstal, 1996), que poderiam interferir na motivação do sujeito em praticar atividades físicas.

Alguns destes pesquisadores usaram o "Inventário de Motivação à Prática Regular de Atividade Física e Esportiva
(IMPRAFE-126)" (Balbinotti \& Barbosa, 2008). Trata-se de um instrumento desenvolvido na realidade brasileira que avalia seis dimensões motivacionais (Controle do Estresse, Saúde, Competitividade, Sociabilidade, Estética e Prazer). Um destes estudos (Barbosa, 2006) incluiu 1377 sujeitos com idade entre 13 e 83 anos. Foram encontradas diferenças significativas entre faixas etárias nas dimensões motivacionais: Saúde, Competitividade, Sociabilidade e Estética, e entre os sexos nas dimensões Controle do Estresse, Saúde e Competitividade. Um segundo trabalho (Balbinotti \& Capozzoli, 2008), realizado com 300 praticantes de atividade física em academia com idades entre 18 e 65 anos, encontrou diferenças significativas nas dimensões motivacionais Controle do Estresse, Competitividade, Sociabilidade e Prazer, quando as faixas etárias foram comparadas, e nas dimensões motivacionais Competitividade e Sociabilidade, quando a comparação foi realizada entre os sexos.

Estudos realizados em outros países e utilizando outros instrumentos também encontraram diferenças entre faixas etárias e sexos, sempre que foram comparadas dimensões motivacionais (Castro, 1999; Lores et al., 2004). Estas evidências empíricas afirmaram a existência de diferenças significativas entre as dimensões motivacionais quando as variáveis sexo e idade são controladas. Cabe saber se estas diferenças persistem quando os níveis gerais de motivação são comparados.

\section{Questão central desta pesquisa}

Partindo-se dos conteúdos teóricos e empíricos apresentados anteriormente, foi possível formular a seguinte questão central desta pesquisa: "existem diferenças significativas nas médias dos escores dos níveis gerais de motivação à prática de atividade física, nos sujeitos investigados, segundo o sexo e a faixa etária dos praticantes?" Para adequadamente responder a esta questão foram empregados procedimentos metodológicos, éticos e estatísticos. Estes procedimentos serão apresentados a seguir.

\section{Método}

\section{Sujeitos}

Uma amostra de 635 praticantes de atividade física regular de ambos os sexos e com idades que variaram de 18 a 55 anos foi selecionada para esse estudo. Salienta-se que esta amostra foi escolhida pelos critérios de disponibilidade e acessibilidade (Maguirre \& Rogers, 1989).

Como se vê na Tabela 1, as idades foram divididas em três grupos, conforme sugerido por Papalia, Olds e Feldman (2006),

Tabela 1

Frequência dos sujeitos por subgrupos da amostra

\begin{tabular}{cccccccc}
\hline \multirow{2}{*}{ Variáveis } & \multicolumn{5}{c}{ Sexo } & \multicolumn{4}{c}{ Grupos de idade (em anos) } \\
\cline { 3 - 7 } & & M & F & 18 a 20 & 21 a 40 & 41 a 55 \\
\hline \multirow{2}{*}{ Sexo } & Masculino & 315 & - & -- & -- & -- \\
& Feminino & -- & 320 & -- & -- & -- \\
\multirow{2}{*}{ Faixa etária } & 18 a 20 & 87 & 47 & 134 & -- & -- \\
(em anos) & 21 a 40 & 202 & 176 & -- & 378 & -- \\
& 41 a 55 & 26 & 97 & -- & -- & 123 \\
\hline
\end{tabular}


a saber: de 18 a 20 anos (adolescente; 21,1\%); de 21 a 40 anos (jovem adulto; 59,5\%); e, de 41 a 55 anos (meia-idade; 19,4\%). Destaca-se, ainda, que esta amostra é composta por $49,6 \%$ de sujeitos do sexo masculino e $50,4 \%$ do sexo feminino.

\section{Instrumentos}

Para a realização da pesquisa foram utilizados dois instrumentos: "Questionário Sócio-Demográfico Simples" (QSDS), apenas para controle das variáveis sexo e idade; "Inventário de Motivação à Prática Regular de Atividade Física e Esporte" (IMPRAFE-126) (Balbinotti \& Barbosa, 2008).

O IMPRAFE-126 é um inventário que avalia seis das possíveis dimensões associadas à motivação à prática regular de atividades físicas. Embora deva ser respondido individualmente, pode ser aplicado a várias pessoas ao mesmo tempo. Tratam-se de 120 itens agrupados 6 a 6 , seguindo a sequência das dimensões a serem estudadas, a saber: controle do stress (por exemplo: "liberar tensões mentais"), saúde (ex.: "manter a forma física"), sociabilidade (ex.: "estar com amigos"), competitividade (ex.: "vencer competições"), estética (ex.: "manter bom aspecto") e prazer (ex.: "meu próprio prazer"). Mais, seis itens que compõem uma escala de verificação: trata-se de itens tomados aleatoriamente (um de cada dimensão) e repetidos no final da escala. A comparação entre as respostas obtidas nos itens em pontos aleatórios da escala e as respostas obtidas na escala de verificação permite avaliar a confiabilidade das respostas.

Responde-se aos itens do IMPRAFE-126 conforme uma escala bidirecional, de tipo Likert, graduada em 5 pontos, indo de "isto me motiva pouquíssimo" (1) a "isto me motiva muitíssimo" (5). O tempo de aplicação é em torno de 20 minutos. As propriedades métricas foram avaliadas (Balbinotti \& Barbosa, 2008) e os resultados indicaram tratar-se de um instrumento válido e fidedigno. Considerando que cada dimensão tem o mesmo número de itens e que as correlações entre as dimensões são todas positivas e altamente significativas ( $r$ de Pearson variou de 0,37 a 0,$69 ; p<0,001)$, pode-se avaliar o nível geral de motivação à prática regular de atividade física somando-se os resultados das seis dimensões do IMPRAFE-126.

\section{Procedimentos de aplicação do Inventário}

Inicialmente, os sujeitos foram contatados nos locais onde são praticadas regularmente atividades físicas (clubes, academias, escolas) para que se pudesse obter o aceite e agendar o dia e o horário da aplicação do Inventário. No dia da aplicação, todos assinaram o Termo de Consentimento Livre e Esclarecido (TCLE), que em seus termos assegurava a confidencialidade das respostas. $\mathrm{O}$ tempo necessário para responder ao IMPRAFE-126 foi de, aproximadamente, 20 minutos. Foram observados os princípios de respeito à pessoa, da beneficência, da não-maleficência (Goldim, 2003) e, ainda, os princípios e regras fundamentais do consentimento informado (Balbinotti \& Wiethaeuper, 2002), todos conforme as diretrizes da Resolução n. 196, de 10 de outubro de 1996, do Conselho Nacional de Saúde. O projeto de pesquisa foi aprovado pelo Comitê de Ética em Pesquisa da Universidade Federal do Rio Grande do Sul (número: 2007721).

\section{Resultados}

A fim de responder, adequadamente, a questão central desta pesquisa, procedeu-se à exploração dos escores obtidos através do IMPRAFE-126, segundo princípios norteadores comumente aceitos na literatura especializada (Bryman \& Cramer, 1999; Pestana \& Gageiro, 2003; Reis, 2001; Trudel \& Antonius, 1991). Sendo assim, apresentam-se, sucessiva e sistematicamente, os resultados das análises de itens, das estatísticas descritivas e das comparações de médias conforme cada variável controlada (sexo, faixa etária). Destaca-se que a apresentação formal da "análise de itens", neste estudo, tem por objetivo demonstrar a confiabilidade dos valores das médias observadas; pois estas, pela possibilidade de sofrer efeitos negativos pela presença de casos extremos (Pestana \& Gageiro, 2003), poderiam não ser representativas dos comportamentos inventariados, diminuindo o valor das conclusões (Balbinotti, 2005).

\section{Análise de Itens}

Destaca-se que as médias encontradas para cada um dos 120 itens estudados individualmente variaram entre 1,73 e 4,51; com desvios-padrões associados variando entre 0,81 a 1,49. Esses resultados indicam que, em média, os jovens respondem as questões do IMPRAFE-126 de forma um pouco mais positiva que negativa. Mesmo assim, interpretam-se esses resultados preliminares como sendo satisfatórios, pois não houve aderência predominante (seja positiva ou negativa) a nenhum dos itens isolados, ou seja, itens com médias semelhantes aos valores extremos (1 ou 5), o que indicaria ausência de variabilidade de respostas - condição que impediria o prosseguimento das análises. Destaca-se, então, que a variabilidade dos resultados indica adequada homogeneidade na dispersão avaliada, diga-se, não houve desvios-padrões com valores nominais maiores que os das médias por item. Já a média encontrada para o instrumento total foi de 385,27, com um desvio-padrão associado de 73,66. Posto que o intervalo total esperado era de 120 a 600 pontos (com média esperada de 360 pontos) e o observado foi de 169 a 592 (com um intervalo interquartil de 93 pontos), observam-se valores próximos entre as médias esperada e observada.

As médias, embora próximas, não são exatamente as mesmas, portanto procedeu-se ao teste $t$ para uma amostra e seus resultados $(t>8,644 ; g l=634 ; p<0,001)$ demonstraram que, embora essas diferenças nominais sejam pequenas, elas foram estatisticamente significativas. Esse resultado vai ao encontro daqueles das médias dos itens e não surpreendem, já que as pessoas que praticam regularmente atividades físicas são, obviamente, motivadas para tal atividade. Ainda, classifica-se a mediana das correlações item-total como moderada $(r=0,46)$, e nenhum índice mostrou-se inferior a 0,20. Estes resultados satisfatórios culminam com os índices Alpha de Cronbach. Os índices Alpha, quando se supõe excluir algum dos 120 itens do inventário, variaram de 0,970 a 0,971. O índice Alpha observado para a escala total (portanto sem a exclusão de nenhum item) é de 0,971. Estes últimos resultados indicam que, efetivamente, todos os itens do questionário contribuem para a consistência interna da medida do construto em estudo. Ainda, considerando que os 120 itens apresentam saturações fatoriais importantes (variando 
de 0,395 a 0,902 , independente da dimensão em estudo) e que todos explicam $51 \%$ da variância total deste construto (Barbosa, 2006), pode-se interpretar, pelo viés da validade de construto, que se está avaliando o que se deseja, ou seja, a motivação à prática regular de atividade física.

\section{Estatísticas descritivas gerais}

Como se pode observar na Tabela 2, os índices obtidos nas médias da motivação (índice geral de motivação), independente da variável controlada, variaram, em valores nominais, moderadamente $(315,15$ a 406,07). Os desvios-padrões seguiram esta mesma ordem, variando de 70,2 a 77,0. Com o objetivo de verificar a adequação do uso de testes paramétricos para a comparação destas médias, e com base nos resultados dos desvios, a igualdade estatística das variâncias foi testada, comprovada e assumida com ajuda do cálculo $F$ de Levène para as variáveis: sexo $\left(F_{(1,633)}=0,334 ; p=0,564\right)$ e faixa etária $\left(F_{(2,632)}=1,119 ; p=0,327\right)$. De maneira geral, esses resultados são excelentes indicadores para o uso de instrumentais paramétricos de comparação das médias. Ainda, conduziu-se o cálculo Kolmogorov-Smirnov, com correção Lilliefors, a fim de testar a aderência à normalidade para a amostra em questão: seus resultados $(K S=0.036 ; g l=635 ; p>0,050)$ também se mostraram satisfatórios $(p>0,05)$.

Tabela 2

Cálculos de tendência central e dispersão por grupo em estudo

\begin{tabular}{cccccc}
\hline \multirow{2}{*}{ Variáveis } & \multicolumn{3}{c}{ Tendência central e dispersão } \\
\cline { 3 - 6 } & & Média & $D P$ & Min & Max \\
\hline \multirow{2}{*}{ Sexo } & Masculino & 385,15 & 74,5 & 206 & 592 \\
& Feminino & 385,38 & 72,9 & 169 & 567 \\
\multirow{4}{*}{ Faixa etária } & $18-20$ & 406,07 & 76,4 & 220 & 592 \\
(em anos) & $21-40$ & 378,24 & 70,2 & 183 & 567 \\
& $41-55$ & 384,20 & 77,0 & 169 & 533 \\
\hline
\end{tabular}

Os valores referentes às estatísticas de dispersão (ver Tabela 2), precisamente quanto aos valores mínimos observados (que variaram de 169 a 592 pontos, independente do grupo em análise), nota-se que existe um relativo afastamento do valor mínimo esperado (que é de 120 pontos). Uma diferença de, no mínimo, 49 pontos. Pode-se dizer que não ocorreu o fenômeno da aquiescência negativa. Isso significa que não houve respondentes inclinados em afirmar que os motivos apresentados no IMPRAFE-126 são, todos, pouquíssimos motivadores para a realização de atividades físicas regulares! Interpretam-se positivamente esses resultados, podendo-se dizer que ninguém da amostra em estudo classifica-se como 'amotivado' à prática regular de atividade física. Sendo assim, e segundo as observações de Brière et al. (1995), pode-se afirmar que a construção motivacional percebida pelos sujeitos investigados encontra-se em níveis satisfatórios. Ou seja, estes indivíduos estão adequadamente aptos a identificar um bom motivo para realizar alguma atividade física regular, indicando que esta lhes trará algum benefício, ou que eles conseguirão realizá-la de modo satisfatório, dentro de seus próprios pontos de vista.

No que se refere aos valores máximos (que variaram de
533 a 592 pontos), destaca-se que houve, no mínimo, um (1) respondente que afirmou sentir-se altamente motivado por quase todos os itens apresentados no IMPRAFE-126. Ao verificar o(s) grupo(s) que ele(s) pertence(m), trata-se de alguém do sexo masculino e da faixa etária entre 18 e 20 anos. Isso, a princípio, poderia ser interpretado como um possível indicador de que manifestações individuais de aquiescência positiva (relativa à motivação à prática regular de atividade física) são mais comumente encontradas em homens desta faixa etária. Outros estudos deveriam testar esta hipótese. Enquanto isso, poder-se-ia pensar que esse resultado está de acordo com aquele de Lores et al. (2004) que afirmaram ser comum em homens desta faixa etária demonstrarem comportamentos mais extremos que as mulheres quando se trata de competitividade e a superação de limites.

Além disso, pode-se ainda acreditar que, na medida em que a faixa etária aumenta, aumenta-se a capacidade discriminatória de respostas dos sujeitos avaliados. Diz-se isso em função do "decréscimo linear na manifestação aquiescente positiva" de respostas ao IMPRAFE-126 (ver valores máximos na Tabela 2). Pode-se interpretar esse resultado considerando que, na medida em que a idade aumenta (ao menos considerando as faixas etárias em estudo), deve existir uma menor probabilidade de aparecerem casos com comportamentos de resposta mais impulsiva. Parece que as pessoas de faixas etárias mais altas discriminam com mais "rigor" quais os motivos que julgam mais positivos e que as levam a praticar uma atividade física regular, assim como aqueles que não as levam. $\mathrm{O}$ ineditismo desta análise não permite comparar esses resultados com os de outros estudos (do construto em questão). Entretanto, conforme um estudo recente (Balbinotti, Wiethaeuper, \& Barbosa, 2004), este comportamento de resposta tem sido observado com outros construtos da personalidade humana, tais como: interesses (gostos e preferências), maturidade (capacidade de enfrentar tarefas de desenvolvimento com as quais se é confrontado, como consequência do próprio desenvolvimento social, biológico e das necessidades da sociedade em relação às pessoas que alcançam este estado de desenvolvimento), e autoconceito (ideias que as pessoas fazem de si mesmo). Sendo assim, futuras ocorrências de casos extremos à direita da curva de distribuição de dados, além de poderem ser interpretadas como sendo características de respostas individuais que, conforme Balbinotti (2005), logicamente podem ocorrer, pode-se também imaginar que alguns comportamentos de resposta, relacionados a certas características da personalidade humana, são mais sensíveis e de resposta mais diferenciada, com o avanço da idade.

Após a apresentação e discussão dos resultados referentes à dispersão dos comportamentos de resposta com a amostra em estudo, apresentam-se e discutem-se os procedimentos e resultados referentes às comparações das médias conforme as variáveis de controle, em estudo.

\section{Comparações de médias por sexo}

Quanto à variável sexo, um teste $t$ para amostras independentes foi conduzido e seus resultados $\left(t_{(633)}=-0,039\right.$; $p=0,969)$ indicaram não existir diferenças significativas ( $p$ $>0,05)$ entre os níveis gerais de motivação à prática regular 
de atividade física. Inicialmente, pode-se dizer que a pequena diferença nominal encontrada nas médias das respostas segundo o sexo dos respondentes não é suficiente para poder-se afirmar que esta variável, quando controlada diretamente, tem um papel importante no construto em questão. Na realidade, seria um tanto surpreendente se um dos sexos se mostrasse mais motivado que o outro. Diga-se, se o fato de pertencer a um ou o outro sexo fosse determinante para se encontrar comportamento de respostas (à motivação) estatisticamente diferentes, seria muito difícil explicar objetivamente tal fenômeno.

Embora homens e mulheres tenham se mostrado igualmente motivados (sob o viés da significância estatística), cabe salientar que ambos os sexos apresentam médias observadas (ver Tabela 2) com cerca de 25 pontos nominais acima da média esperada (360 pontos). A fim de se testar os níveis de significância para essas diferenças, realizou-se uma comparação, com a ajuda do teste $t$ para uma amostra, que indicou tratar-se de índices de motivação estatisticamente iguais ao esperado para o instrumento, tanto para os homens $\left(t_{(314)}=0,036 ; p>0,971\right)$ quanto para as mulheres $\left(t_{(319)}=0,093 ; p>0,926\right)$. Interpretam-se esses resultados de forma positiva, pois este pode ser um dos indicadores de que, em média, os sujeitos que compuseram esta amostra em estudo não estão expressando a intenção iminente de abandonar a prática regular de atividade física. As vantagens de tal decisão afetam diretamente a saúde geral desses sujeitos, o que tem sido observado na literatura nacional (Balbinotti \& Capozzoli, 2008; Graça \& Bento, 1992) e internacional (Capdevila, Niñerola, \& Pintanel, 2004; Morgan \& Goldston, 1987; Wankel, 1993). Por outro lado, pode-se imaginar a possibilidade de que o instrumento tenha sido construído de forma a favorecer respostas equilibradas, mas isso só poderá ser verificado com pesquisas continuadas a partir de diversas amostras independentes. Assim, espera-se que outros estudos, com outras amostras, testem e discutam estas propriedades métricas, verificando se este comportamento de resposta se repete.

Considerando esses resultados referentes às comparações estatísticas gerais em relação ao sexo, pode-se ficar inclinado a supor que, possivelmente, não existam diferenças entre as dimensões constitutivas do construto em estudo (Motivação à Prática Regular de Atividade Física), mas isso não deve ser suposto. Mesmo que não seja um dos objetivos deste estudo a descrição das médias por dimensão constitutiva desta motivação, cabe dizer que essas diferenças podem ocorrer e, se assim for o caso, novos estudos devem apresentá-las e discuti-las, efetiva e sistematicamente.

Na verdade, alguns estudos têm avaliado as diferenças entre as dimensões da motivação. Por exemplo, o estudo realizado por Lores et al. (2004) testou a existência de possíveis diferenças entre homens e mulheres praticantes de atividade física na dimensão estética. Seus resultados indicam que as mulheres são significativamente $(p<0,001)$ mais motivadas por aspectos relacionados à estética, do que os homens. Em contrapartida, o mesmo estudo demonstrou que os homens são significativamente $(p<0,001)$ mais motivados do que as mulheres quando a dimensão avaliada é a competitividade. Resultados similares foram encontrados por Balbinotti e Capozzoli (2008). Sendo assim, parece que, em média, as dimensões se acomodam de forma que o índice geral da motivação resta indiferente entre homens e mulheres.

\section{Comparações de médias por faixa etária}

Quanto à variável faixa etária, realizou-se a estatística ANOVA one-way e seus resultados $\left(F_{(2,632)}=7,214 ; p=0,001\right)$ indicaram existir ao menos uma diferença altamente significativa $(p>0,01)$ entre as médias observadas nas três faixas etárias, em estudo. O teste complementar de Bonferroni (considerando que as variâncias são homogêneas) permitiu localizar duas diferenças significativas $(p<0,05)$. Uma delas, altamente significativa $(p<$ $0,01)$, foi localizada entre as médias do grupo de adolescentes (18 a 20 anos) e do grupo de jovens adultos (21 a 40 anos); e outra diferença significativa $(p<0,05)$, localizada entre as médias do grupo de adolescentes (18 a 20 anos) e do grupo de meia idade (41 a 55 anos); ambas favoráveis aos adolescentes (ver Tabela 2).

O comportamento do índice geral de motivação ao longo das idades (considerando os três grupos estudados) pode ser simbolicamente representado por uma letra "V", com a segunda "perna" um pouco mais curta (ver Figura 1). Estes resultados podem ser discutidos tanto à luz da teoria da autodeterminação, quanto à luz da teoria do desenvolvimento humano.

No que diz respeito à teoria da autodeterminação, os elevados índices de motivação entre os adolescentes observados neste estudo podem ser interpretados como um indicador de que a motivação destes sujeitos é predominante intrínseca. Esta interpretação é sustentada por achados teóricos e empíricos que indicam que níveis elevados de motivação estão associados à motivação intrínseca (Ryan et al., 1997).

No que diz respeito à teoria do desenvolvimento humano, pode se pensar que os adolescentes (18 a 20 anos) enfrentam tarefas de desenvolvimento que incluem a exploração e teste das potencialidades e limites de seus corpos, que, diga-se de passagem, estão em pleno processo de transformação (Aberastury \& Knobel, 1981). As atividades físicas, por um lado, propiciam a exploração deste corpo, testando seus limites e, por outro lado, favorecem o aprendizado e seu condicionamento geral; que conduzem à realização de novas tarefas, reiniciando este processo, de caráter dinâmico.

Ainda sob o mesmo viés (da teoria do desenvolvimento humano), outros aspectos poderiam contribuir para explicar os altos índices de motivação na adolescência, tais como o aumento e a diversificação de atividades sociais nesta fase. Segundo Weinberg e Gould (2001), as atividades físicas, de maneira geral, favorecem a socialização, o que contribui na transposição das tarefas desta fase. Esta explicação é reforçada pelos resultados de estudos como aquele realizado por Balbinotti e Capozzoli (2008), onde se viu que os mais jovens são significativamente $(p<0,05)$ mais motivados por aspectos ligados à socialização.

Quanto à redução dos índices de motivação observada na segunda faixa etária (jovens adultos, 21 a 40 anos), podese pensar que, teoricamente, quando estes sujeitos entram na vida adulta, as tarefas próprias da adolescência (aprendizado, experimentação, exploração do corpo) estão concluídas ou em fase de conclusão. As tarefas típicas dos adultos jovens são as de estabelecimento e avanço profissional - estabilização em uma profissão, consolidação do papel de trabalhador e interesse 


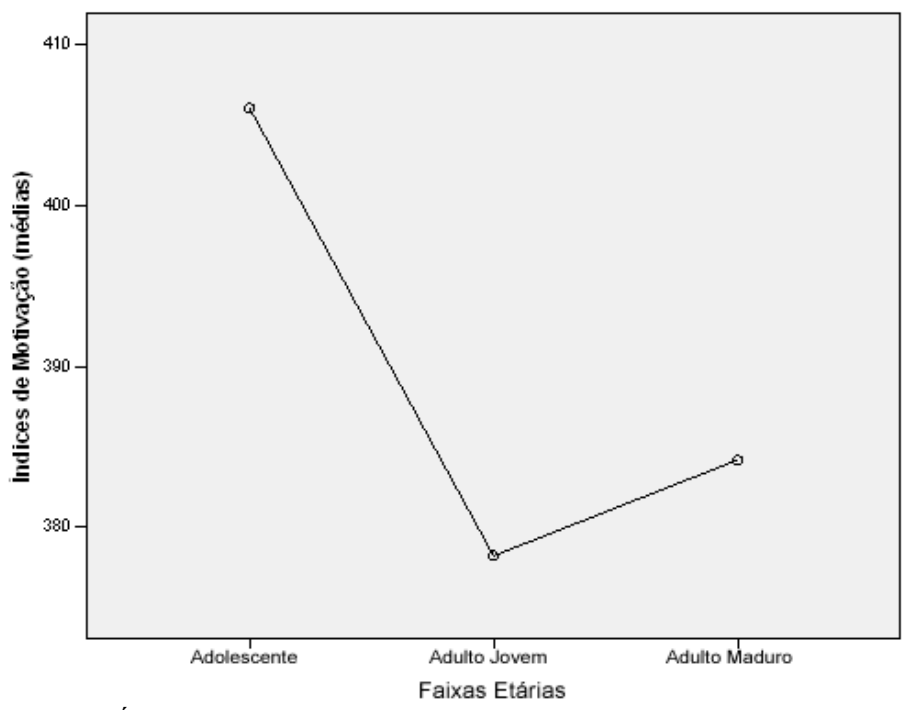

Figura 1. Índice geral de Motivação à prática de atividade física nas faixas

eminente de avançar profissionalmente (Balbinotti, 2003) - e a assunção de novas responsabilidades - relacionamentos, casamento, filhos, etc. (Papalia et al., 2006). Sendo assim, a redução dos índices de motivação à prática de atividade física observada nos adultos jovens pode ser interpretada como decorrente do tipo de tarefa de desenvolvimento, ou seja, na vida adulta as tarefas são bastante menos relacionadas ao corpo do que aquelas (já mencionadas) da adolescência.

Quanto a terceira faixa etária (meia idade, 41 a 55 anos), observa-se uma elevação nominal nos índices de motivação a prática de atividade física, quando comparado com o grupo de jovens adultos (21 a 40 anos). Esta elevação nos valores nominais (elevação não significativa, $p>0,05$ ) também pode ser interpretada pelo viés da teoria do desenvolvimento.

Como se sabe, esta fase tem relativamente poucas tarefas novas. Desde o início da década de 1960, Super, Starishevsky, Matlin e Jordaan (1963), já indicavam que, no que diz respeito ao âmbito profissional, trata-se de uma faixa etária onde uma das principais tarefas associadas é a de manter a posição profissional. Esta fase é denominada como de 'manutenção', indicando que as principais tarefas são manter a posição, atualizar-se e inovarse. São tarefas de adaptação e preservação do autoconceito. As atividades físicas permitem, ao menos em parte, responder as demandas desta fase, já que a adoção de uma prática de atividade física, frequentemente, tem sido associada ao conceito de juventude, disposição, energia (Garcia \& Lemos, 2003) e pode ser vista como um jeito de se preservar, atualizar e inovar.

Outro viés para a compreensão desta elevação nominal dos índices de motivação na meia idade são os resultados de estudos como os de Balbinotti e Capozzoli (2008) e Barbosa (2006), que indicam que a motivação relacionada à saúde e ao abandono do sedentarismo aumenta significativamente com o passar dos anos. Na verdade, a percepção de que a saúde é um bem que deve ser preservado e conquistado por meio de comportamentos e hábitos saudáveis ao longo da vida, tem sido sustentada por diferentes estudos (Morgan \& Goldston, 1987; Paffenbarger, Hyde, \& Wing, 1990; Wankel, 1993) e as pessoas parecem estar cada vez mais conscientes disso. Estas evidências têm incentivado a prática de atividades físicas regulares com vistas à manutenção da saúde e a prevenção de doenças associadas ao sedentarismo (Capdevila et al., 2004). Sendo assim, se pode entender que a motivação encontrada nesta faixa etária é predominantemente extrínseca (Ryan et al., 1997).

\section{Conclusões, limitações e perspectivas para novos estudos}

Este trabalho permitiu explorar diferenças e semelhanças estatísticas encontradas nas respostas de 635 praticantes regulares de atividade física, de ambos os sexos, com idades variando de 18 a 55 anos. A partir dos cuidados nos procedimentos éticos e metodológicos, os resultados indicam não haver diferenças significativas nas respostas dos sujeitos quando controlada a variável sexo. Mesmo não sendo possível generalizar os resultados obtidos nesta pesquisa, pois o procedimento de coleta amostral foi não-aleatório, tanto o número de sujeitos em estudo quanto a pluralidade setorial onde foram coletadas as respostas dos sujeitos permitem algumas conclusões hipotéticas importantes. Mesmo considerando esta limitação de caráter amostral, pode-se concluir que o fato de pertencer a qualquer um dos sexos não afeta os índices gerais de motivação à prática regular de atividade física.

No que diz respeito às idades, os resultados indicaram haver ao menos uma diferença significativa entre os índices gerais de motivação dos sujeitos quando a variável faixa etária é controlada. Teste complementar permitiu localizar diferenças significativas entre as faixas etárias, precisamente entre o grupo de adolescentes e os grupos de adultos jovens e de meia-idade (favorável ao grupo de adolescentes em ambos os casos). Os índices mais elevados dos adolescentes foram entendidos como decorrentes do tipo dos tipos de tarefas de desenvolvimento próprias desta fase e do tipo de motivação (intrínseca) que poderia mover estes adolescentes. Os menores índices observados nos demais grupos foram entendidos como sendo decorrentes das mudanças na natureza das tarefas de desenvolvimento próprias de cada fase e do tipo de motivação (extrínseca). 
Cabe finalizar este estudo indicando que estes resultados podem ser particularmente úteis para psicólogos do esporte, professores de Educação Física, personal trainers e outros profissionais interessados por assuntos relacionados à atividade física e exercício com adolescentes e adultos (de 18 a 55 anos), e que também se interessam em explorar seus níveis de motivação à prática regular de atividade física. Então, dimensões como "controle de estresse", "saúde", "sociabilidade", "competitividade", "estética" e "prazer" parecem ser uma fonte importante de informação para esses profissionais, permitindo que eles entendam melhor como esses elementos se integram na dinâmica geral de funcionamento daqueles que se beneficiam. Destaca-se que estes profissionais devem considerar as diferenças estatisticamente significativas aqui apresentadas e criar modelos de orientação e de educação à prática regular de atividades físicas que sejam adequados aos grupos específicos estudados. Medidas de motivação ao exercício são particularmente interessantes quando utilizadas dentro de um contexto maior, ou seja, quando esses profissionais se interessam em ajudar as pessoas na preparação de uma vida integral.

Novos estudos devem ser conduzidos a fim de se verificar a existência de diferenças estatísticas nos níveis de motivação à prática de atividade física, mas controlando mais de uma variável em conjunto - metodologias multivariadas de análise - (por exemplo, mulheres de 21 a 40 anos em relação a homens de 21 a 40 anos, controlados pelo tipo de atividade física). Assim, acredita-se possível melhor explicar os fenômenos relativos a esse importante aspecto da atividade humana e de seu desenvolvimento. Assim, sugere-se a realização de outros estudos com um intervalo maior de idades, abarcando, inclusive, outras variáveis dependentes importantes (cidades - capital e interior -, áreas urbanas, semi-rurais e rurais, entre outras) bem como a inclusão de outras etapas (fases) do desenvolvimento humano.

\section{Referências}

Aberastury, A., \& Knobel, M. (1981). Adolescência normal. Porto Alegre: Artes Médicas.

Balbinotti, M. A. A. (2003). A noção transcultural de maturidade vocacional na teoria de Donald Super. Psicologia: Reflexão e Crítica, 16(3), 461-473.

Balbinotti, M. A. A. (2005). Para se avaliar o que se espera: reflexões acerca da validade dos testes psicológicos. Aletheia, 1(21), 43-52.

Balbinotti, M. A. A., \& Barbosa, M. L. L. (2008). Análise da consistência interna e fatorial confirmatória do IMPRAFE-126 com praticantes de atividades físicas gaúchos. Psico-USF, 13(1), 1-12.

Balbinotti, M. A. A., \& Capozzoli, C. J. (2008). Motivação à prática regular de atividade física: um estudo exploratório com praticantes em academias de ginástica. Revista Brasileira de Educação Física e Esporte, 22(1), 63-80.

Balbinotti, M. A. A., \& Wietheauper, D. (2002). Princípios e regras fundamentais do consentimento informado: uma proposta de intervenção em psicologia. Revista Fahrenheit 451, 2(3), 48-67.

Balbinotti, M. A. A., Wiethaeuper, D., \& Barbosa, M. L. L. (2004). Níveis de cristalização de preferências profissionais em alunos de ensino médio. Revista Brasileira de Orientação Profissional, 5(1), 15-28.

Barbosa, M. L. L. (2006). Propriedades métricas do Inventário de Motivação para a Prática Regular de Atividade Física (IMPRAF-126) (Dissertação de mestrado não-publicada). Universidade Federal do Rio Grande do Sul, Porto Alegre.
Brière, N., Vallerand, R., Blais, M., \& Pelletier, L. (1995). Developpement et validation d'une mesure de motivation intrinséque, extrinséque et d'amotivation en contexte sportif: l'echelle de motivation dam les sports. International Journal of Sport Psychology, 26(4), 465-489.

Bryman, A., \& Cramer, D. (1999). Quantitative data analysis: a guide for social scientists. New York: Routledge Edition.

Capdevila, L., Niñerola, J., \& Pintanel, M. (2004). Motivación y actividad física: el autoinforme de motivos para la práctica de ejercicio físico (AMPEF). Revista de Psicología del Deporte, 13(1), 55-74.

Castro, S. I. S. (1999). As danças tradicionais portuguesas como actividade de recreação e lazer: motivação para a prática dos Grupos de Etnografia e Folclore do Douro Litoral. (Dissertação de mestrado não-publicada). Faculdade de Ciências do Desporto e de Educação Física da Universidade do Porto, Portugal.

Conselho Nacional de Saúde. (1996). Resolução CNS 196/96. Recuperado de http://www.extranet.ceuma.br/downloads_2007/pesquisa/comite_resolucao. pdf

Deci, E. L., \& Olson, B.C. (1989). Motivation and competition: their role in sports. In J. H. Goldstein (Org.), Sports, games, and play (2 ${ }^{\mathrm{a}}$ ed., pp. 83110). Hillsdale: Erlbaum.

Deci, E. L., \& Ryan, R. M. (1985). Intrinsic motivation and self-determination in human behavior. New York: Plenum.

Deci, E. L., Ryan, R. M., \& Koestner, R. (1999). A meta-analytic review of experiments examining the effects of extrinsic rewards on intrinsic motivation. Psychological Bulletin, 125(6), 627-668.

Fernandes, H. M., \& Vasconcelos-Raposo, J. (2005). Continuum de AutoDeterminação: validade para a sua aplicação no contexto desportivo. Estudos de Psicologia, 10(3), 385-395.

Frederick, C. M., \& Ryan, R. M. (1995). Self-determination in sport: a review using cognitive evaluation theory. International Journal of Sport Psychology, $26,5-23$.

Garcia, R. P., \& Lemos, K. M. (2003). A Estética como um valor na Educação Física. Revista Paulista de Educação Física, 17(1), 32-40.

Goldim, J. R. (2003). Princípios Éticos. Recuperado de http://www.bioetica. ufrgs.br/princip.htm.

Graça, A., \& Bento, J. B. (1993). Receios e convicções de controlo acerca da saúde em crianças e jovens. In A. Marques (Org.), A ciência do desporto, a cultura e o homem: $3^{\circ}$ Congresso de Educação Física dos Países de Língua Portuguesa (pp. 599-612). Porto: Faculdade de Desporto da Universidade do Porto.

Lores, A., Murcia, J., Sanmartín, M., \& Camacho, Á. (2004). Motivos de práctica físico-desportiva según la edad y el género en una muestra de universitarios. Apunts, 76, 13-21.

Maguirre, T. O., \& Rogers W. T. (1989). Proposed solutions for non randomness in educational research. Canadian Journal of Education, 14(2), 170-181.

Morgan, W. P., \& Goldston, S. E. (1987). Exercise and mental health. Washington: Hemisphere.

Paffenbarger, R. S., Hyde, R. T., \& Wing, A. L. (1990). Physical activity and physical fitness as determinants of health and longevity. In C. Bouchard, R. J. Shephard, T. Stephens, J. R. Sutton, \& B. D. McPherson (Orgs.), Exercise, fitness, and health: a consensus of current knowledge (pp. 33-48). Champaign: Human Kinetics.

Papalia, D. E., Olds, S. W., \& Feldman, R. D. (2006). Desenvolvimento humano ( $8^{\mathrm{a}}$ ed). Porto Alegre: Artmed.

Pestana, M. H., \& Gageiro, J. G. (2003). Análise de dados para ciências sociais. a complementaridade do SPSS ( $3^{\underline{a}}$ ed.). Lisboa: Edições Silabo.

Reis, E. (2001). Estatística multivariada aplicada (2 $2^{\mathrm{a}}$ ed.). Lisboa: Edições Silabo.

Ryan, R. M., \& Deci, E. L. (2000a). Intrinsic and extrinsic motivations: classic definitions and new directions. Contemporary Educational Psychology, 25(1), 54-67.

Ryan, R. M., \& Deci, E. L. (2000b). Self-Determination Theory and the facilitation of intrinsic motivation, social development, and well-being. American Psychologist, 55(1), 68-78. 
Ryan, R. M., Frederick, C. M., Lepes, D., Rubio, N., \& Sheldon, K. M. (1997). Intrinsic motivation and exercise adherence. International Journal of Sport Psychology, 28, 335-354.

Super, D. E., Starishevsky, R., Matlin, N., \& Jordaan, J. P. (1963). Career development: self-concept theory. New York: College Entrance Examination Board.

Trudel, R., \& Antonius, R. (1991). Métodes quantitatives appliquées aux sciences humaines. Montréal: Les Éditions de la Chenelière.
Wang, J., \& Wiese-Bjornstal, D. M. (1996). The Relationship of school type and gender to motives for sport participation among youth in the people's republic of China. International Journal of Sport Pshycology, 28, 13-24.

Wankel, L. M. (1993). The importance of enjoyment to adherence and psychological benefits from physical activity. International Journal of Sport Psychology, 24, 151-169.

Weinberg, R. S., \& Gould, D. (2001). Fundamentos da psicologia do esporte e do exercício ( $2^{\mathrm{a}}$ ed.). Porto Alegre: Artmed.

Marcos Alencar Abaide Balbinotti, PhD em psicologia e pós-doutor pela Universidade de Montreal-Canadá, pós-doutor pela Universidade de Sherbrooke- Canadá, é professor da Université du Québec à Trois-Rivières. Endereço para correspondência: Université du Québec à Trois-Rivières, Département de psychologie, 3351, boul. des Forges, C.P. 500, Trois-Rivières (QC), G9A 5H7. Tel.: (819)376-5011 ramal 4058.Fax: (819)3765210. E-mail: marcos.balbinotti@uqtr.ca

Marcus Levi Lopes Barbosa, mestre em Ciências do Movimento Humano pela Universidade Federal do Rio Grande do Sul, é doutorando no Programa de Pós-graduação em Ciências do Movimento Humano da Universidade Federal do Rio Grande do Sul. E-mail: marcus_barbosa@yahoo.com Carlos Adelar Abaide Balbinotti, doutor em Ciências do Desporto pela Faculdade do Desporto da Universidade do Porto-Portugal, é professor Adjunto IV da Escola de Educação Física da Universidade Federal do Rio Grande do Sul. E-mail: cbalbinotti@terra.com.br Ricardo Pedrozo Saldanha, mestre em Ciências do Movimento Humano pela Universidade Federal do Rio Grande do Sul, é professor da Faculdade Cenecista de Osório-RS. E-mail: ricardo@ricardosaldanha.com.br 